\title{
Learning to count: adapting population monitoring for Endangered huemul deer Hippocamelus bisulcus to meet conservation objectives
}

\author{
Heiko U. Witt mer, Pa ulo Corti, Cristián Sa ucedo and José Luis Galaz
}

\begin{abstract}
Considerable efforts have been invested in recent years to improve methods for both data collection and analyses required for population monitoring. Where historical or current estimates of population size are not adjusted for detection probabilities they may be too inaccurate to provide meaningful estimates of trends and thus monitoring methods need to be adapted. Here, we use data from the Endangered huemul deer Hippocamelus bisulcus to outline a framework to develop accurate robust estimates of detection probabilities that can be incorporated into new surveys in a cost-effective way and applied to existing survey data sets. In particular, by retroactively estimating detection probabilities for surveys of huemul, we show that current survey methods for huemul are inadequate to determine population trends reliably. Based on these results we propose a new monitoring method for the huemul and discuss the importance of estimating accuracies of historical survey data to ensure that changes in the abundance of the species reflect real population trends and are not an artefact of variation over time in the accuracy of survey data.
\end{abstract}

Keywords Abundance, Chile, Hippocamelus bisulcus, huemul, matrix model, monitoring, Patagonia, ungulate

\section{Introduction}

Strategies for the conservation of threatened populations $S$ are predicated on the ability to assess population size accurately and detect changes in abundance (Yoccoz et al., 2001; Lancia et al., 2005). As counting all individuals in a population is generally infeasible, sampling regimes must be designed to produce precise unbiased estimates of abundance and population parameters. Considerable efforts have

\footnotetext{
Heiko U. WittmeR* (Corresponding author) Wildlife, Fish, and Conservation Biology, University of California, Davis, California, USA. E-mail heiko. wittmer@vuw.ac.nz

Paulo Corti Instituto de Zoología, Universidad Austral de Chile, Valdivia, Chile

Cristián Saucedo Conservación Patagónica, Patagonia Park, Cochrane, Chile

José Luis Galaz Gerencia Áreas Protegidas y Medio Ambiente, Corporación Nacional Forestal, Santiago, Chile

${ }^{*}$ Current address: School of Biological Sciences, Victoria University of Wellington, P.O. Box 600, Wellington 6140, New Zealand

Received 22 August 2009. Revision requested 5 October 2009.

Accepted 6 November 2009.
}

been invested in recent years to improve methods for both data collection and analyses required for population monitoring. In particular, recent methodological advances have helped to address problems associated with imperfect detection of many species (Yoccoz et al., 2001; Williams et al., 2002; Tyre et al., 2003; Lancia et al., 2005). Unfortunately, estimating detection probabilities for many species is expensive and time consuming, and those charged with the management of threatened taxa are understandably hesitant to adopt costly new methods unless their benefits have been clearly demonstrated. In addition, the adoption of new methods may invalidate comparisons between current and historical estimates of population size unless detection probabilities can be estimated retroactively. Therefore, there is a need to develop accurate robust estimates of detection probabilities that can be incorporated into new surveys in a cost-effective way and applied to existing survey data sets.

Huemul Hippocamelus bisulcus are medium-size deer restricted to southern Chile and Argentina. Historically, the species was considered abundant across a range of habitats from central Chile $\left(34^{\circ} \mathrm{S}\right)$ to the Magellan Strait $\left(53^{\circ} \mathrm{S}\right.$; Cabrera \& Yepes, 1960). Since the arrival of Europeans the range of the huemul has been reduced by $>50 \%$ (Vila et al., 2006) and it now occurs almost exclusively in the montane forests of the southern Andes and along Chile's southern coast. This range reduction has been accompanied by a dramatic reduction in abundance. Estimates indicate that $<2,000$ remain, probably a $99 \%$ reduction from estimated historical abundances (Redford \& Eisenberg, 1992). Because of the low remaining population size, declining population trends and the risks associated with increased population fragmentation, the species is categorized as Endangered on the IUCN Red List (Jiménez et al., 2008).

Despite its precarious conservation status, reliable information on status and population trends of huemul is scarce. The virtual absence of population monitoring is probably a consequence of both inadequate funding and biological characteristics that make monitoring the huemul challenging. For example, huemul live in small groups, mainly in forested areas (Frid, 1999; Corti, 2008). Both these factors have been shown to pose challenges for accurate population monitoring in other species (Morellet et al., 2007; McConville et al., 2009). In addition, remaining populations of huemul are often restricted to remote and inaccessible habitats (such as periglacial areas and coastal forests), making fieldwork a particular challenge. 
In areas where huemul are being monitored population estimates are based on survey methods that are not adjusted for detection probabilities (CONAF, 2001). Consequently, survey results are presented with no measure of uncertainty, which means that the accuracy of these population estimates is unknown. The uncertainty of current population estimates is troubling because existing monitoring programmes may not be sufficiently accurate to detect trends that may have important consequences for huemul conservation, management and policies. In addition, it may prevent researchers from diagnosing causes of population fluctuations.

Our objectives are three-fold. Firstly, we aim to determine the accuracy of the survey method currently used in the only long-term monitoring programme for huemul in Chile. To achieve this, we present results from population surveys of huemul conducted by the Chilean Forest Service (Corporación Nacional Forestal; CONAF) during $1991-2008$ and use these results to estimate annual finite rates of increase $(\lambda)$ for the population. We then compare these results to complete retroactive population counts based on marked individuals available for 2005-2008. During this time all adults of the population were individually identifiable, which allowed us to estimate the accuracy of the surveys conducted by CONAF. Secondly, we use population models to evaluate the likelihood that year-to-year variation in estimates of population size from the CONAF surveys could be explained by natural process variation (i.e. reflecting true variability in vital rates based on environmental conditions). Thirdly, we propose a survey framework for huemul, based on capture-recapture methods, which could be used to monitor populations of huemul across their remaining range. Such monitoring is essential to develop effective recovery strategies for this species. Our proposed framework may be applicable to other species of conservation concern where past monitoring did not consider detection probabilities.

\section{Study area}

Our study was conducted in the Lago Cochrane National Reserve, Aysén District, Chilean Patagonia (Fig. 1). This $69.25 \mathrm{~km}^{2}$ forest reserve was established in 1967 , c. $4 \mathrm{~km}$ east of the town of Cochrane (3,000 inhabitants). Forest vegetation is dominated by deciduous lenga Nothofagus pumilio trees at higher elevations and the evergreen coigüe Nothofagus dombeyi at lower elevations, with introduced pine (Pinus spp.) trees patchily distributed throughout the Reserve. The shrub layer primarily consists of ñirre Nothofagus antarctica, and a variety of grasses and forbs occur in open habitats as a consequence of human-induced fires in the 1940s. The terrain in the Reserve is mostly mountainous, with elevations of $150-1,100 \mathrm{~m}$. The mean annual temperature is $7.6^{\circ} \mathrm{C}$. Mean annual precipitation is c. $800 \mathrm{~mm}$, the majority of which falls during the winter (June-September). Extensive sheep farming (small family farms to medium-size operations) is the most common land use in the areas surrounding the Reserve.

\section{Methods}

\section{Population monitoring}

Huemul monitoring in the Lago Cochrane National Reserve was initiated by CONAF in 1984 and provides the only long-term monitoring data for this species in Chile. During the initial years monitoring focused primarily on fawn surveys. Beginning in 1991 CONAF conducted systematic annual surveys of the entire huemul population in the Reserve. Since 1991 a total of 17 annual surveys have been conducted. Surveys were carried out by CONAF personnel and usually occurred either during autumn (March-April, $\mathrm{n}=14$ ) or just prior to the fawning season (October-November, $\mathrm{n}=3$ ) when huemul are typically found at lower elevations (Gill et al., 2008).

In general, multiple observers (the number was 20-30 in any given year) walked along predetermined elevational contour lines, with one person assigned to every 20-50 m of altitude. Observations focused on areas at $150-700 \mathrm{~m}$ and observers recorded both direct (visual) and indirect (tracks and faeces) observations of huemul. In addition, information on age (adults, juveniles and fawns) and sex of encountered huemul was also recorded. Surveys were conducted over 2 consecutive days and because direct observations of the same individual by different observers during the same day were considered unlikely, only the results of the higher tally were used for final population estimates. From these survey data we estimated $\lambda$ adjusted for annual time intervals. We restricted our calculations of $\lambda$ to direct observations of adults because in species with high variability in fawn survival (e.g. many ungulates; Gaillard et al., 2000), variation in the size of the adult breeding population may be a more meaningful indicator for determination of long-term population trends.

Starting in 2005 we initiated an intensive monitoring project that included identifying all individual huemul. This project ran parallel to the CONAF surveys and covered the same population. The goal of our monitoring project was to mark every huemul in the Reserve to obtain data on population size, trends and the demographic rates determining the observed trends (Corti et al., 2010). Between April 2005 and November 2008 we captured and marked a total of 55 huemul of all age and sex classes with VHF radio-collars $(n=15)$, VHF radio-ear tags $(n=18)$ and global positioning system collars $(\mathrm{n}=3)$. Additional animals, including individuals with and without telemetry 


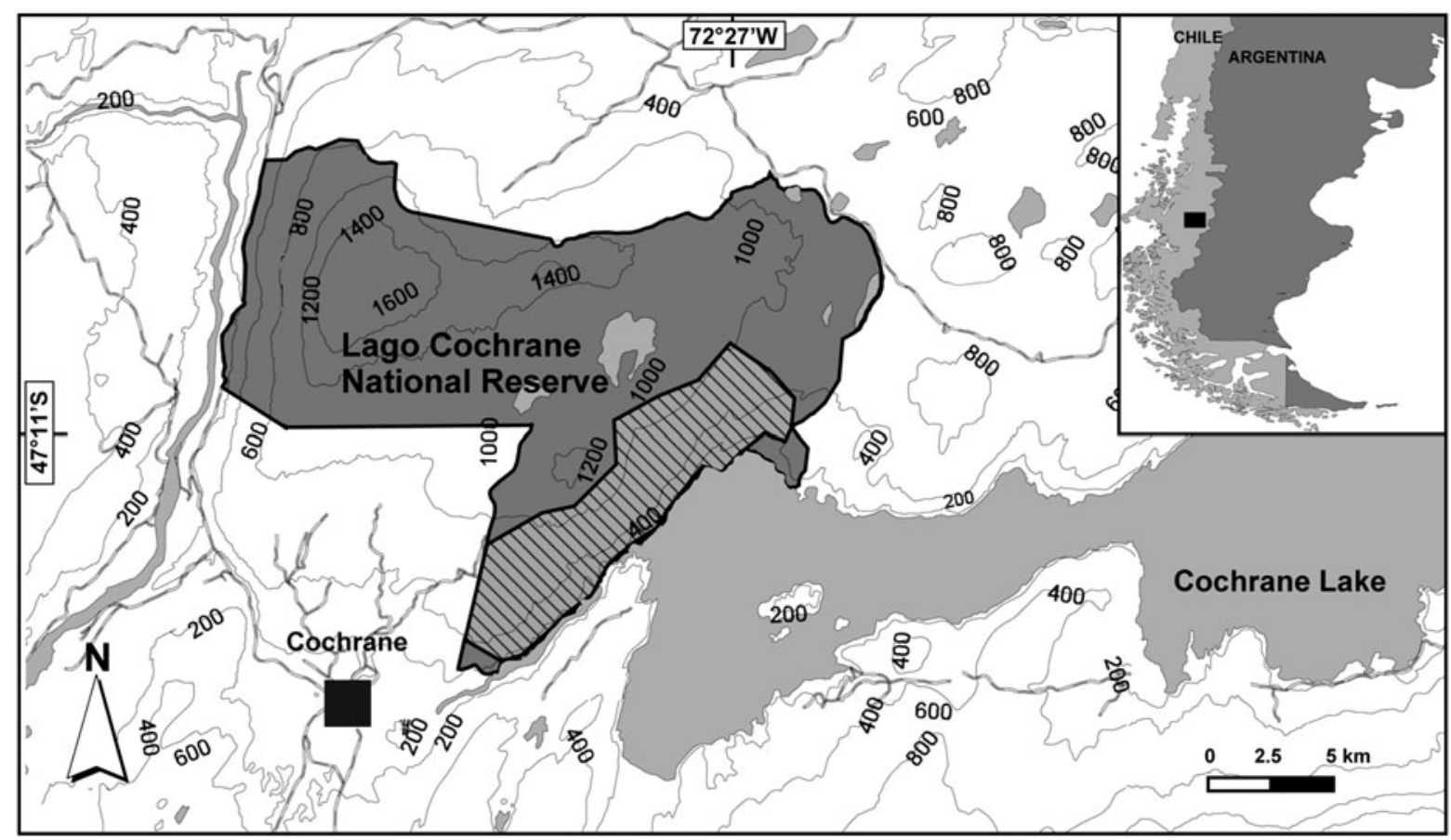

FIG. 1 The study area for huemul Hippocamelus bisulcus in Lago Cochrane National Reserve, Aysén District (reserve indicated in dark grey). The approximate range of the survey area is shown with grey hatching (modified from Corti et al., 2010). The black rectangle on the inset indicates the location of the main figure in Chilean Patagonia.

devices, received conventional ear tags $(\mathrm{n}=37)$. We were also able to identify 33 additional huemul based on their natural markings (e.g. facial patterns, body shape, coat colour and rump patch) or their association with marked individuals. Our goal of identifying all individuals was facilitated by two unique aspects of our study population. Firstly, in the absence of widespread hunting or poaching, huemul are generally easy to approach. Secondly, the study population is currently restricted to a small $\left(19.9 \mathrm{~km}^{2}\right)$ part of the Reserve (Corti et al., 2010).

The presence of all known individuals was confirmed by direct observation at least once every 3 weeks. Marked individuals were considered dead if we found their remains (59\%) or if we were unable to relocate known individuals alive for the remainder of the study (41\%). In general the majority of individuals that died were found because of the large sample of radio-collared animals and because scavenging birds helped with the detection of other individuals that died (Corti et al., 2010). Of animals with unknown fates, only two were classified as adults. In addition, we monitored the Reserve at regular intervals to detect animals that may have been missed during previous surveys or that immigrated into the Reserve during our study. Based on the encounter history of all individuals we then retroactively estimated the number of adult individuals in the Reserve in November of each year, just prior to parturition (i.e. prebreeding population size; sensu Caswell, 2001). Consecutive population estimates were used to determine $\lambda$. Comparing results of the two different monitoring programmes al- lowed us to retroactively estimate the detection probability of the CONAF monitoring programme.

\section{Population modelling}

We used a matrix modelling approach (Caswell, 2001) to evaluate the likelihood that estimated year-to-year variation in $\lambda$ from the CONAF monitoring programme reflected true variability in vital rates (i.e. process variation). Survival rates for huemul in the Reserve were estimated for four age classes (fawns, yearlings, 2 year-olds and adults older than 3 years) by Corti et al. (2010) using a Kaplan-Meier approach (Pollock et al., 1989). Additionally, fertility rates were estimated directly from observations of marked females with a fawn (Corti et al., 2010). Because no female younger than 3 years of age was observed with a fawn at heel, we assumed age of primiparity to be 3 years. We then used mean values for age-specific survival and fertility rates (Table 1) to parameterize the following (pre-breeding) population matrix:

$$
A=\left[\begin{array}{ccc}
0 & 0 & F_{3+} * p_{0} \\
p_{1} & 0 & 0 \\
0 & p_{2} & p_{3+}
\end{array}\right],
$$

where $F_{3^{+}}$is adult female fertility (i.e. 0.72$)$ and $p_{\mathrm{x}}$ represents the age-specific survival probabilities of fawns $\left(p_{0}=\right.$ $0.34)$, yearlings $\left(p_{1}=0.88\right), 2$ year-olds $\left(p_{2}=0.91\right)$ and adult females $\left(p_{3^{+}}=0.91\right)$. Assuming a stable age distribution $\lambda$ can 
TABLE 1 Estimates of fertility and age-specific survival rates for huemul Hippocamelus bisulcus in Lago Cochrane National Reserve (Fig. 1; from Corti et al., 2010) used to parameterize matrix models. ${ }^{1}$

\begin{tabular}{lllll}
\hline Vital rate & Mean & Low $(\%)$ & Average $(\%)$ & Large (\%) \\
\hline Fertility $\left(F_{3+}\right)$ & 0.72 & \pm 10 & \pm 15 & \pm 20 \\
Fawn survival $\left(p_{0}\right)$ & 0.34 & \pm 30 & \pm 40 & \pm 50 \\
Yearling survival $\left(p_{1}\right)$ & 0.88 & \pm 10 & \pm 15 & \pm 20 \\
Juvenile survival $\left(p_{2}\right)$ & 0.91 & \pm 5 & \pm 10 & \pm 15 \\
Adult survival $\left(p_{3+}\right)$ & $0.91^{2}$ & \pm 5 & \pm 10 & \pm 15 \\
\hline
\end{tabular}

${ }^{1}$ The three models simulate the effects of low, average and large year-toyear process variation on population growth rates $(\lambda)$

${ }^{2}$ Recalculated from Corti et al. (2010) to include both females and males

then be estimated from the dominant eigenvalue of the projection matrix (Caswell, 2001).

We then incorporated estimates of year-to-year process variation associated with mean values of survival and fertility into the matrix model following Wittmer et al. (2007). This approach required sampling both survival and fertility values from specified probability distributions. Matrix models were performed using Excel (Microsoft, Redmond, USA) and the Excel add-in PopTools (Hood, 2005). Values for all vital rates were selected using the Excel function NORMINV(probability, mean and standard_dev), where probability is a uniform random number from $o$ to 1 (selected using RAND()), and mean and standard_dev were the estimated values for vital rates and likely process variation, respectively. Each set of randomly selected vital rates for the population was then used to construct a timeinvariant matrix population model. We repeated the procedure 1,000 times, re-sampling from the probability distribution of each vital rate for each replicate.

We based estimates of process variation for vital rates on assumptions outlined in Gaillard et al. (2000), suggesting that most ungulates generally show low year-to-year variability in adult survival (generally $<10 \%$ ), medium variability in both yearling survival and adult fertility (generally $<15 \%$ ) and high variability in fawn survival (often $\geq 40 \%$ ). From these assumptions, we built three different models that varied in their assumptions of likely process variation (Table 1). Confidence intervals for $\lambda$ obtained from the repeated samples were determined using the percentile method (Efron \& Tibshirani, 1993).

\section{Results}

Uncorrected population surveys conducted by CONAF over 1991-2008 indicated that the Lago Cochrane National Reserve population varied between 14 adults in 1991 and 50 adults, observed both in 1996 and in 1997 (Table 2). Adult sex ratios observed during counts were biased towards females $($ mean $=0.51 \pm$ SD 0.07 ).
TABLe 2 Numbers of male, female and adult huemul counted in population surveys conducted by CONAF in Lago Cochrane National Reserve (Fig. 1) during 1991-2008, with female-to-male ratio and estimates of the annual finite rate of increase $(\lambda)$.

\begin{tabular}{lcclll}
\hline Year & Males & Females & Adults & F/M ratio & $\lambda$ \\
\hline 1991 & 7 & 7 & 14 & 0.50 & \\
1993 & 7 & 9 & 16 & 0.56 & $1.07^{*}$ \\
1994 & 13 & 22 & 35 & 0.63 & 2.19 \\
1995 & 24 & 23 & 47 & 0.49 & 1.34 \\
1996 & 23 & 27 & 50 & 0.54 & 1.06 \\
1997 & 28 & 22 & 50 & 0.44 & 1.00 \\
1998 & 9 & 9 & 18 & 0.50 & 0.36 \\
1999 & 20 & 18 & 38 & 0.47 & 2.11 \\
2000 & 26 & 23 & 49 & 0.47 & 1.29 \\
2001 & 17 & 19 & 36 & 0.53 & 0.73 \\
2002 & 13 & 14 & 27 & 0.52 & 0.75 \\
2003 & 14 & 12 & 26 & 0.46 & 0.96 \\
2004 & 21 & 24 & 45 & 0.53 & 1.73 \\
2005 & 21 & 21 & 42 & 0.50 & 0.93 \\
2006 & 7 & 11 & 18 & 0.61 & 0.43 \\
2007 & 14 & 14 & 28 & 0.50 & 1.56 \\
2008 & 8 & 9 & 17 & 0.53 & 0.61 \\
\hline
\end{tabular}

${ }^{*}$ Adjusted for 1-year time interval

Annual estimates of $\lambda$ of the huemul breeding population from uncorrected population surveys in the Reserve varied (0.36-2.19; Table 2). Population trends were positive during 8 years, unchanged during 1 year and negative (i.e. $\lambda<1.0)$ during 7 years. Since $2000 \lambda$ was mostly negative $(\mathrm{n}=6)$. In 1994 and $1999 \lambda$ indicated that the breeding population was more than double the previous survey. Eleven of the 16 estimates of $\lambda$ between consecutive years indicated either catastrophic population declines $(\lambda \leq 0.75$, $\mathrm{n}=5)$ or very high population increases $(\lambda \geq 1.25, \mathrm{n}=6)$.

Based on our encounter histories of marked individuals we were able to retroactively estimate the population size during 4 different years. Population estimates varied between 23 adults in 2005 and 34 adults in 2008 (Table 3). Sex ratios during all years were strongly biased towards females (mean $=0.62 \pm 0.03)$.

TABLE 3 Retroactive estimates of the numbers of male, female and adult huemul in Lago Cochrane National Reserve (Fig. 1), based on individually identified animals during 2005-2008, with female-to-male ratio and estimates of the finite rate of increase $(\lambda){ }^{1}$

\begin{tabular}{lcllll}
\hline Year & Males & Females & Adults & F/M ratio & $\lambda$ \\
\hline 2005 & 9 & 14 & $23^{2}$ & 0.61 & \\
2006 & $13^{3}$ & 19 & 32 & 0.59 & \\
2007 & 10 & 20 & 30 & 0.67 & 0.94 \\
2008 & 13 & 21 & 34 & 0.62 & 1.13 \\
\hline
\end{tabular}

${ }^{1} \lambda$ not calculated for $2005-2006$ because not all individuals had been identified in 2005

${ }^{2}$ Not all individuals had been identified in the first sampling period

${ }^{3}$ Adjusted to include one male first observed in February 2007 


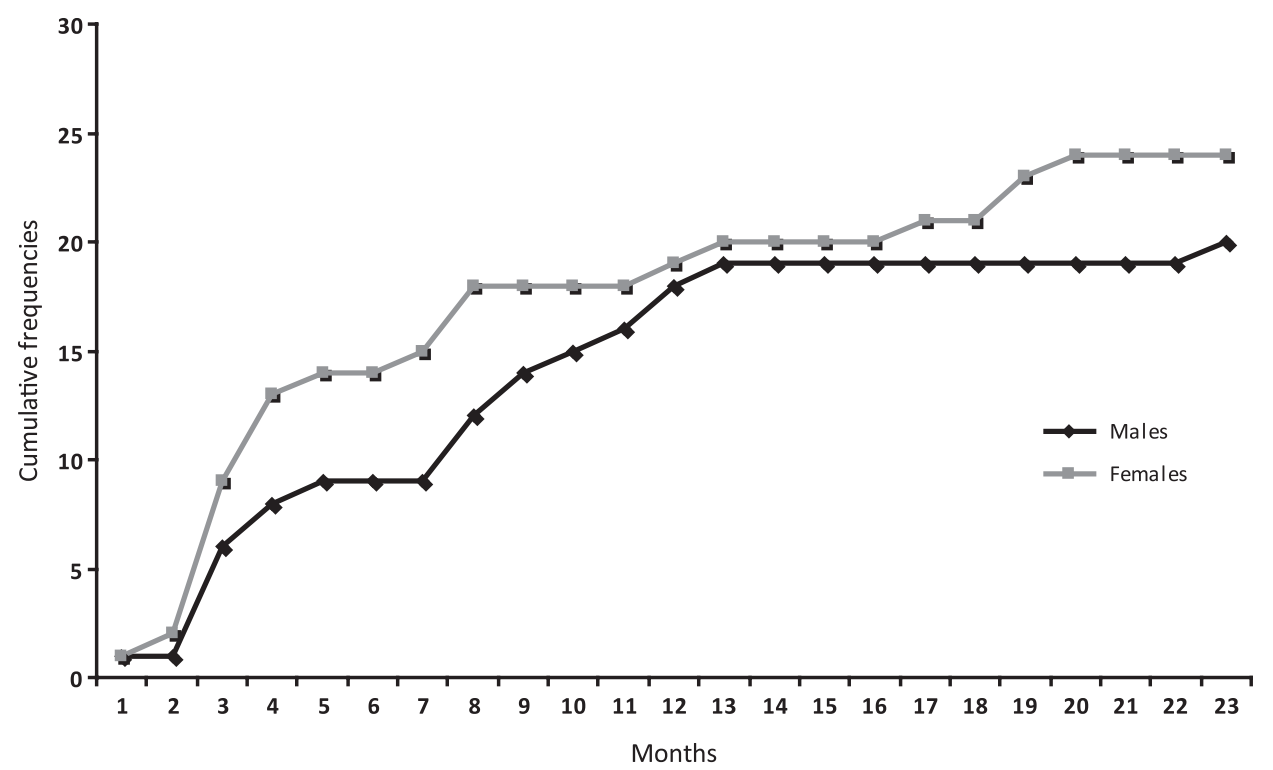

FIG. 2 Cumulative monthly frequencies of individually identifiable huemul at Lago Cochrane National Reserve (Fig. 1) since monitoring began in April 2005. All individuals were identifiable/marked by February 2007 (Month 23 of the study).

Intensive surveys starting in April 2005 indicated that it took until February 2007 (23 months) before all adult huemul believed to be present at the beginning of our monitoring were individually identifiable (Fig. 2). Thus, $\lambda$ was only estimated for 2006-2008 (Table 3). Additional individuals transitioning to the adult age class during the study were first known during earlier stages of their lives.

In the 3 years where both corrected and uncorrected survey data were available (i.e. 2006-2008), uncorrected data consistently underestimated actual population sizes of huemul in the area. The detection probability during these years averaged $67 \pm$ SD $23 \%$. Estimates of $\lambda$ and sex ratios of adult huemul also differed markedly between the two methods.

Estimates of mean $\lambda$ from the matrix models for all three scenarios were 1.06-1.08 (Fig. 3). Even under the most generous assumptions of expected process variation affecting vital rates, 11 of the 16 estimates of $\lambda$ from the CONAF surveys (Table 2) fall outside identified 95\% confidence intervals.

\section{Discussion}

Our results highlight a problem relevant for many species of conservation concern. The development and implementation of successful recovery strategies for threatened species depends to a significant degree on reliable estimates of population size and trends. However, many survey methods, including those used by CONAF for huemul, do not accurately determine the status of a population. As in other species (Williams et al., 2002), including ungulates (Largo et al., 2008), our results also indicate that these problems can be overcome by adapting monitoring of huemul through using survey methods that allow quantifying detection probabilities based on individually marked animals.

Population surveys conducted by CONAF during 20062008 that did not account for detection probabilities consistently underestimated the total population size of huemul in the Lago Cochrane National Reserve. While on average $31 \%$ of adults in the study area remained undetected

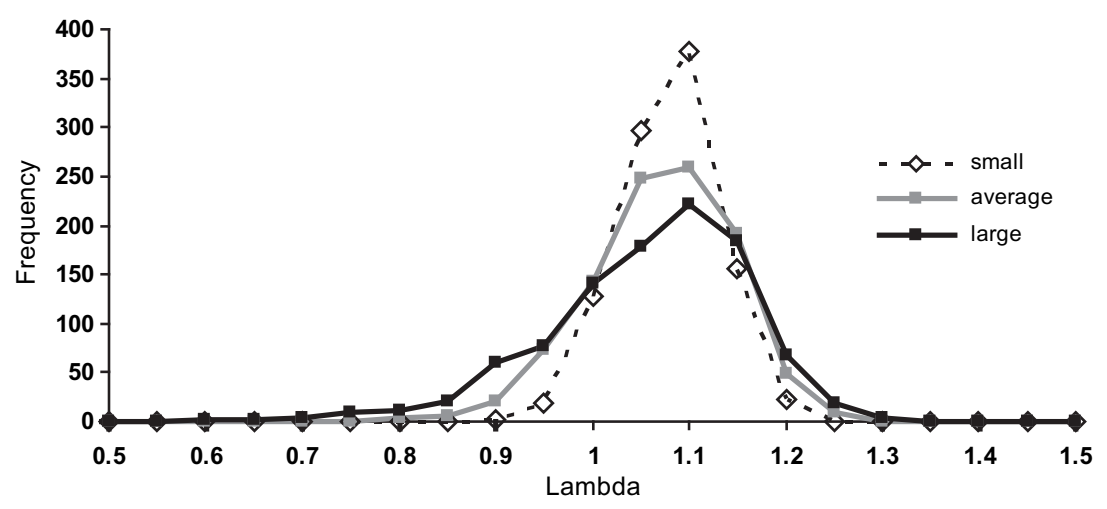

FIG. 3 Frequency distributions of the annual finite rate of increase $(\lambda)$ for three different matrices calculated from 1,000 repeated random measurements of $\lambda$, assuming three different levels of process variation (see Table 1 for numerical values of process variation). 
during CONAF's annual surveys, this estimate is probably conservative because in some years juvenile animals $(<3$ years old) were probably misclassified as adults. Detection probabilities apparently also varied greatly among years. The sampling error associated with some population surveys for huemul was so large that it suggested population fluctuations of a magnitude that are impossible for monotocous ungulates with life history strategies such as those of huemul (Gaillard et al., 2000). The ability to detect real changes in population size in threatened species, however, is essential for the development of both short- and longterm conservation strategies.

Accurate information regarding population size and observed and projected trends are also important criteria for the IUCN Red List (Mace et al., 2008). The current categorization of huemul as Endangered is based on estimates suggesting a total remaining population of $<2,000$ individuals, approximately two-thirds of which occur in Chile (Jiménez et al., 2008). However, this value may not reflect the real abundance of the species because estimates for most individual populations were not based on survey data (Vila et al., 2006). The absence of accurate population estimates makes the task of determining current or anticipated population trends across the remaining distribution of huemul virtually impossible. Thus, future reassessments of the status of huemul have to carefully consider if reported changes in the abundance of the species reflect real population trends or are potentially an artefact of more accurate survey data. This would avoid unwarranted changes to the assessment of its conservation status (i.e. avoid downgrading of threatened status where not warranted).

Several ecological and behavioural characteristics contribute to the difficulties of accurately determining the abundance of huemul. Firstly, huemul live in mostly forested and mountainous habitats. Because of poor visibility and steep terrain, counting animals in such habitat is challenging. Secondly, remaining huemul populations occur at low densities and in small groups. Reported densities for the species are typically c. $1 \mathrm{~km}^{-2}$ (Smith-Flueck \& Flueck, 1997; Corti et al., 2010). Thirdly, huemul typically occur in groups of $<_{3}$ (Frid, 1999; Corti, 2008), with no sexual segregation observed during any time of the year (Corti, 2008). Species occurring in such small groups are particularly difficult to detect.

However, results from our intensive monitoring programme suggest that accurate population estimates of huemul using direct counts are possible. This requires a change in the protocol of how huemul abundances are estimated. In particular, our results show the value of maintaining a sample of marked individuals. A large sample of marked individuals allowed us to identify outliers in the CONAF survey results (see Largo et al., 2008, for an example with European ibex) as well as to quantify detection probabilities for the species. Marking can be achieved by applying ear tags or radio-collars to individuals. While ear tags are cheaper, telemetry studies provide additional benefits such as the ability to quantify agespecific survival as well as determining causes of mortality (Corti et al., 2010). Encounter history data can then be used to estimate population size using standard capture-markrecapture methods (Williams et al., 2002). Park wardens charged with huemul conservation can also create databases with photos of huemul to distinguish individuals based on visual characteristics. However, such an approach would probably be more cost and labour intensive, and less accurate in the long term, than marking animals with tags or radio-collars. Priority for new monitoring programmes should be given to populations in other areas (such as coastal Patagonia) to test whether our proposed method can be applied across the remaining distribution of huemul (and across different habitats).

Our results have highlighted the need to quantify detection probabilities when survey data are being used to estimate population trends. Many survey methods currently employed produce estimates of population size and trends that are biased in ways similar to those reported here. For example, aerial surveys are commonly used to estimate abundances of large mammals in open habitats (Caughley, 1974; Marsh et al., 2004). Recent results reported for saiga Saiga tatarica indicated that reported rates of decline for the species were overestimated because the detection probability of groups from the air changed with declining population densities (McConville et al., 2009). In particular, saiga occur in smaller groups at low population densities and these groups were less likely to be detected during aerial surveys. Such biases in detection probability have at least two important implications. Firstly, it raises questions regarding results of studies aiming to link rates of decline to ecological or behavioural explanations of these trends (Milner-Gulland et al., 2003). Secondly, it requires re-evaluating conservation targets to avoid downgrading of the conservation status of a species when it remains at risk of extinction. As with our study, such examples highlight the importance of estimating accuracies of historical survey data to ensure that changes in the abundance of a species reflect real population trends and are not an artefact of variation over time in the accuracy of survey data.

\section{Acknowledgements}

We thank the Denver Zoological Foundation, Conservación Patagónica, the National Geographic Society and Idea Wild for financial support, and all people who helped in the capturing of huemul and in the fieldwork. PC was supported through a Natural Science and Engineering Research Council of Canada grant to Marco Festa-Bianchet. CONAF Aysén District Office granted access to the Lago Cochrane National Reserve. Capture permits for huemul 
were issued by the Wildlife Subdepartment, Natural Renewable Resources Division of the Agricultural Service of Chile (Servicio Agrícola y Ganadero). We thank A. Marshal, J.-M. Gaillard, D. Shackleton and an anonymous reviewer for helpful comments on earlier versions of this article.

\section{References}

Cabrera, A. \& Yepes, J. (1960) Mamíferos Sudamericanos, 2nd edition. Edian, Buenos Aires, Argentina.

Caswell, H. (2001) Matrix Population Models: Construction, Analysis, and Interpretation, 2nd edition. Sinauer Associates, Sunderland, USA.

Caughley, G. (1974) Bias in aerial survey. Journal of Wildlife Management, 38, 921-933.

CONAF (Corporación Nacional Forestal) (2001) Plan para la conservación del huemul del sur Hippocamelus bisulcus en Chile. Unpublished Report. Corporación Nacional Forestal, Santiago, Chile.

Corti, P. (2008) Organisation sociale, dynamique de population, et conservation du cerf huemul (Hippocamelus bisulcus) dans la Patagonie du Chili. PhD thesis, Université de Sherbrooke, Quebec, Canada.

Corti, P., Wittmer, H.U. \& Festa-Bianchet, M. (2010) Dynamics of a small population of endangered huemul deer (Hippocamelus bisulcus) in Chilean Patagonia. Journal of Mammalogy, 91, 690-697.

Efron, B. \& Tibshirani, R. (1993) An Introduction to the Bootstrap. Chapman \& Hall, London, UK.

Frid, A. (1999) Huemul (Hippocamelus bisulcus) sociality at a periglacial site: sexual aggregation and habitat effects of group size. Canadian Journal of Zoology, 77, 1083-1091.

Gaillard, J.M., Festa-Bianchet, M., Yoccoz, N.G., Loison, A. \& Toigo, C. (2000) Temporal variation in fitness components and population dynamics of large herbivores. Annual Review in Ecology and Systematics, 31, 367-393.

Gill, R., Saucedo, C., Aldridge, D. \& Morgan, G. (2008) Ranging behaviour of huemul in relation to habitat and landscape. Journal of Zoology, 274, 254-260.

Hood, G.M. (2005) Pop Tools (version 2.6.6). Http://www.cse.cisro. au/poptools [accessed 1 August 2009].

Jiménez, J., Guineo, G., Corti, P., Smith, J.A., Flueck, W., Vila, A. et al. (2008) Hippocamelus bisulcus. In IUCN Red List of Threatened Species v. 2010.2. Http://www.iucnredlist.org [accessed 6 July 2010].

Lancia, R.A., Kendall, W.L., Pollock, K.H. \& Nichols, J.D. (2005) Estimating the number of animals in wildlife populations. In Techniques for Wildife Investigations and Management, 6th edition. (ed. C.E. Braun), pp. 106-153. The Wildlife Society, Bethesda, USA.

Largo, E., Gaillard, J.-M., Festa-Bianchet, M., Toïgo, C., Bassano, B., Cовтот, H. et al. (2008) Can ground counts reliably monitor ibex Capra ibex populations? Wildlife Biology, 14, 489-499.

Mace, G.M., Collar, N.J., Gaston, K.J., Hilton-Taylor, C., AкÇAKaya, H.R., Leader-Williams, N. et al. (2008) Quantification of extinction risk: IUCN's system for classifying threatened species. Conservation Biology, 22, 1424-1442.
Marsh, H., Lawler, I.R., Kwan, D., Delean, S., Pollock, K. \& Alldredge, M. (2004) Aerial surveys and the potential biological removal technique indicate that the Torres Strait dugong fishery is unsustainable. Animal Conservation, 7, 435-443.

McConville, A.J., Grachev, I.A., Keane, A., Coulson, T., Bekenov, A.B. \& Milner-Gulland, E.J. (2009) Reconstructing the observation process to correct for changing detection probability of a Critically Endangered species. Endangered Species Research, 6, 223-229.

Milner-Gulland, E.J., Bukreeva, O.M., Coulson, T.N., Lushchekina, A.A., Kholodova, M.V., Bekenov, A.B. \& GracheV, I.A. (2003) Reproductive collapse in saiga antelope harems. Nature, 422, 135.

Morellet, N., Gaillard, J.-M., Hewison, A.J.M., Ballon, P., Boscardin, Y., DUnCAN, P. et al. (2007) Indicators of ecological change: new tools for managing populations of large herbivores. Journal of Applied Ecology, 44, 634-643.

Pollock, K.H., Winterstein, S.R., Bunck, C.M. \& Curtis, P.D. (1989) Survival analysis in telemetry studies: the staggered entry design. Journal of Wildlife Management, 53, 7-15.

Redford, K.H. \& EisenberG, J.F. (1992) Mammals of the Neotropics, Vol. 2. The Southern Cone: Chile, Argentina, Uruguay, Paraguay. University of Chicago Press, Chicago, USA.

Smith-Flueck, J.A.M. \& Flueck, W.T. (1997) Relevamiento de una población de huemul en la provincia de Río Negro, Argentina. Mastozoologia Neotropical, 4, 25-33.

Tyre, A.J., Tenhumberg, B., Field, S.A., Niejalke, D., Parris, K. \& Possingham, H.P. (2003) Improving precision and reducing bias in biological surveys: estimating false-negative error rates. Ecological Applications, 13, 1790-1801.

Vila, A.R., López, R., Pastore, H., Faúndez, R. \& Serret, A. (2006) Current distribution and conservation of the huemul (Hippocamelus bisulcus) in Argentina and Chile. Mastozoologia Neotropical, 13, 263-269.

Williams, B.K., Nichols, J.D. \& Conroy, M.J. (2002) Analysis and Management of Animal Populations: Modeling, Estimation, and Decision Making. Academic Press, San Diego, USA.

Wittmer, H.U., Powell, R.A. \& King, C.M. (2007) Understanding contributions of cohort effects to growth rates of fluctuating populations. Journal of Animal Ecology, 76, 946-956.

Yoccoz, N., Nichols, J.D. \& Bouliner, T. (2001) Monitoring of biological diversity in space and time. Trends in Ecology \& Evolution, 16, 446-453.

\section{Biographical sketches}

Heiko U. Wittmer's research interests focus on the ecology and conservation of mammals, primarily ungulates, in multi-prey, multipredator systems. He is a member of the IUCN Deer Specialist Group. PAUlo CORTI researches behavioural and molecular ecology of large mammals and birds and evolutionary processes affecting wildlife conservation. CRISTIÁN SAUCEDo is the wildlife manager for Conservación Patagónica and oversees the conversion of the former Estancia Chacabuco into the future Patagonia National Park. José Luis Galaz is the Director of the Natural Protected Areas Service for CONAF, Chile. He has worked on research and management of natural resources in Chile, Peru, Bolivia, Spain and Italy. 